\title{
Distribution spatiale de deux monogènes du genre Protoancylodiscoides Paperna, 1969 (Dactylogyridea, Ancyrocephalidae) parasites branchiaux de Chrysichthys auratus (Geoffroy Saint-Hilaire, 1808) (Siluriformes, Claroteidae) dans le Lac Ossa (Littoral -Cameroun)
}

\author{
Nack Jacques ${ }^{* 1,}$, Massende Josephine ${ }^{2}$ et Messu Mandeng D. Françoise ${ }^{3}$ \\ 1 Institut de Sciences Halieutiques, Université de Douala, BP7236 Douala, Cameroun \\ 2 Département de Biologie des Organismes Animaux, Faculté de Sciences, Université de Douala BP 24157 Douala, \\ Cameroun. jennynobel2014@yahoo.fr \\ 3 University of Yaoundé I, Higher Teacher Training College, P.O. Box 47 Yaoundé, Cameroon. \\ messumandeng@yahoo.fr \\ *Corresponding author email : jacquesnack@yahoo.fr
}

Original submitted in on $6^{\text {th }}$ November 2017. Published online at www.m.elewa.org on $31^{\text {st }}$ January 2018 https://dx.doi.org/10.4314/jab.v121i1.6

\section{RESUME}

Objectifs : Le but de cette étude a été d'investiguer sur le mode de répartition de Protoancylodiscoides combesi et $P$. auratum monogènes parasites branchiaux de Chrysichthys auratus (Siluriformes, Claroteidae), communément appelé machoiron, un poisson abondamment exploité dans l'ensemble du bassin de la Sanaga, afin de déterminer les mécanismes qui influencent leur fixation.

Méthodologie et résultats: les poissons ont été pêchés au filet maillant d'avril en juin 2016, conservés dans des bocaux contenant du formol puis disséqués au laboratoire pour isoler, monter et identifier les monogènes parasites. II est apparu que les deux espèces de monogènes sont fréquentes et peu fréquentes respectivement (prévalence $=71,4 \%$ et 28,60 ), leurs abondances sont faibles et très faibles (Intensité moyenne $=34,14$ et 16, 37 respectivement). Les deux monogènes montrent une distribution agrégative. Ils exploitent les côtés gauche et droit, les individus mâles et femelles de la même manière. Leur gradient de fixation sur les arcs branchiaux se fait dans le sens antéro-postérieur, c'est-à-dire de l'arc I vers l'arc IV. Les deux vers fréquentent prioritairement le secteur médian, quoique leurs charges soient similaires sur les trois secteurs.

Conclusion et application des résultats : Ce travail suggère une fois de plus que les espèces du genre Protoancylodiscoides ont un modèle de fixation antéro-postérieur sur les arcs branchiaux et qu'en situation de faible intensité leur fixation sur les secteurs des arcs se fait au hasard. En général les charges parasitaires sont très faibles ou faible ce qui suggère que l'élevage de $C$. auratus doit se faire dans un environnement peu modifié

Mots clés : monogènes, écologie, Protoancylodiscoides, Chrisichthys auratus, Cameroun. 
Spatial distribution of two monogeneans of the genus Protoancylodiscoides Paperna, 1969 (Dactylogyridea, Ancyrocephalidae), gill parasites of Chrysichthys auratus (Geoffroy Saint-Hilaire, 1808) (Siluriformes, Claroteidae) in Lake Ossa (Littoral -Cameroon)

\begin{abstract}
Objectives: The aim of this study was to investigate the distribution of Protoancylodiscoides combesi and $P$. auratum monogeneans gill parasites of Chrysichthys auratus (Siluriformes, Claroteidae), commonly called machoiron, a widely exploited fish throughout the Sanaga Basin, and determine the mechanisms that influence their fixation.

Methodology and Results: fish were caught using gillnets from April to June 2016, stored in jars containing formalin and dissected in the laboratory to isolate, mount and identify monogeneans species. Both species of monogeneans were found to be frequent and less frequent, respectively (prevalence $=71.4 \%$ and 28.60 ), their abundances are low and very low (mean intensity $=34,14$ and 16, 37 respectively). The two monogeneans show an aggregative distribution. They exploited the left and right sides, male and female individuals in the same way. Their fixation gradient on the gill arches is in the antero-posterior direction, from arch I to the arch IV. The two worms mainly attend the median sector, although their loads are similar in the three gill arch sectors.

Conclusion and application of results: this work suggests once again that Protoancylodiscoides species have an antero-posterior fixation model on gill arches and, in a low intensity situation; their fixation on gill arches sectors is random. In general, parasite loads are very low or low suggesting that $C$. auratus breeding should be done in a poorly modified environment
\end{abstract}

Key words: monogeneans, ecology, Protoancylodiscoides, Chrisichthys auratus, Cameroon.

\section{INTRODUCTION}

La production mondiale de la pêche de capture en 2014 a été de 93,4 millions de tonnes dont 81,5 millions de tonnes prélevées en mer et 11,9 millions de tonnes dans les eaux continentales (FAO, 2016). La contribution de la pêche continentale est donc importante. Le même rapport indique par ailleurs que l'offre mondiale du poisson en 2014 a atteint le chiffre record de $20 \mathrm{~kg}$ par habitant par an, à la faveur de l'aquaculture qui fournit désormais la moitié du poisson destiné à la consommation humaine (FAO, 2016). La production aquacole mondiale en 2014 a ainsi atteint $44,1 \%$ de la production mondiale contre $42,1 \%$ en 2012 et $37 \%$ en 2004 (FAO, 2016) En Afrique subsaharienne, le poisson couvre en moyenne un peu plus de $22 \%$ des apports en protéines d'origine animale. Cette couverture serait proche de $50 \%$ dans certains pays côtiers comme le Cameroun (Anonyme, 2000). L'Afrique connait actuellement une crise de la disponibilité de poissons qui est essentiellement due à la stagnation des prises alors que la population continue d'augmenter (Worldfish center, 2005). En 2012 le Cameroun a produit 12052 tonnes de poissons. II a importé la même année 118609 tonnes de poisson frais et de conserves, pour une valeur estimée à 68,5 milliards de FCFA (INS, 2013). Le développement de l'aquaculture permettrait donc au Cameroun de faire d'énormes bénéfices. Cette activité devrait se faire avec des espèces autochtones à bonne résilience. Plusieurs travaux ont permis d'identifier au sein de l'ordre des Siluriformes plusieurs espèces présentant un fort potentiel en aquaculture. Chrysichthys auratus est une espèce abondamment pêchée et commercialisée dans la Réserve de Faune du Lac Ossa et dans d'autres rivières et fleuves du Cameroun. II présente un fort potentiel pour l'aquaculture du fait de sa taille considérable (Iongueur totale maximale : $267 \mathrm{~mm}$ ) ce qui suppose une production protéique importante pour chaque individu. (Stiassny et al., 2007). En situation d'élevage, on assiste souvent à des épizooties pouvant nuire la reproduction et la santé des poissons. Ces parasites provoquent aussi grâce à leur crochets et leurs ventouses des difficultés respiratoires, des lésions importantes et la sécrétion du mucus pouvant entrainer la mort (Obiekezie et al., 1988; Okaeme et al., 1988). Le genre Chrysichthys 
est répandu dans 16 pays de l'Afrique (Angola, Benin, Cameroun, Côte d'Ivoire, Égypte, Gabon, Gambie, Ghana, Liberia, Niger, Nigéria ; République Démocratique du Congo Sénégal, Sierra Leone, Tchad et Togo). Au total 8 espèces de Monogènes ont été décrites chez Chrysicthys spp), il s'agit de Proancylodiscoides chrysicthes Paperna, 1969 chez Chrysichthys nigroditatus au Ghana; Protoancylodiscoides mansourensis El-naggar, 1987 chez Chrysichthys auratus en Égypte (El-Naggar, 1987) Protoancylodiscoides combesi Bassock, Nack et Pariselle, 2016 et Protoancylodiscoides auratum Bassock, Nack et Pariselle, 2016 au Cameroun (Bassock et al., 2016); Protoancylodiscoides sp1

\section{MATÉRIEL ET MÉTHODES}

Site d'étude: Chrysichthys auratus (Pisces, Claroteidae), espèce hôte étudiée au cours de ce travail, est un poisson qui peut atteindre $27 \mathrm{~cm}$ de longueur totale (Stiassny et al., 2007). Les spécimens ont été capturés de nuit au filet maillant dans le Lac Ossa (fig. 1), chez C. auratus, Chrysichthys maurus et C. nigrodigitatus; Protoancylodiscoides sp2 chez $C$. maurus et $C$. nigrodigitatus et Protoancylodiscoides sp3 chez C. nigrdigitatus (Euzet et al., 1989). Jusqu'à présent, l'écologie des monogènes du genre Protoancylodiscoides n'a été abordée que par Ahmed et al. (2003) en Égypte sur le modèle C. auratus/P. mansourensis. Au Cameroun, les études sur l'écologie des monogènes parasites de ce poisson n'ont pas encore été faites. L'objectif de cette étude est donc d'analyser la distribution spatiale de ces deux parasites chez $C$. auratus ainsi que les mécanismes qui déterminent leur fixation.

affluent du Fleuve Sanaga. Ce plan d'eau est formé de trois lacs naturels interconnectés (Lac Ossa le plus vaste, Lac Mevia et Lac Mvembé), tous situés dans la localité de Dizangué $\left(3^{\circ} 45^{\prime} 42^{\prime \prime}\right.$ et $3^{\circ} 53^{\prime}$ de latitude Nord et entre 9०9' et $10^{\circ} 4^{\prime} 12^{\prime \prime}$ de longitude Est.

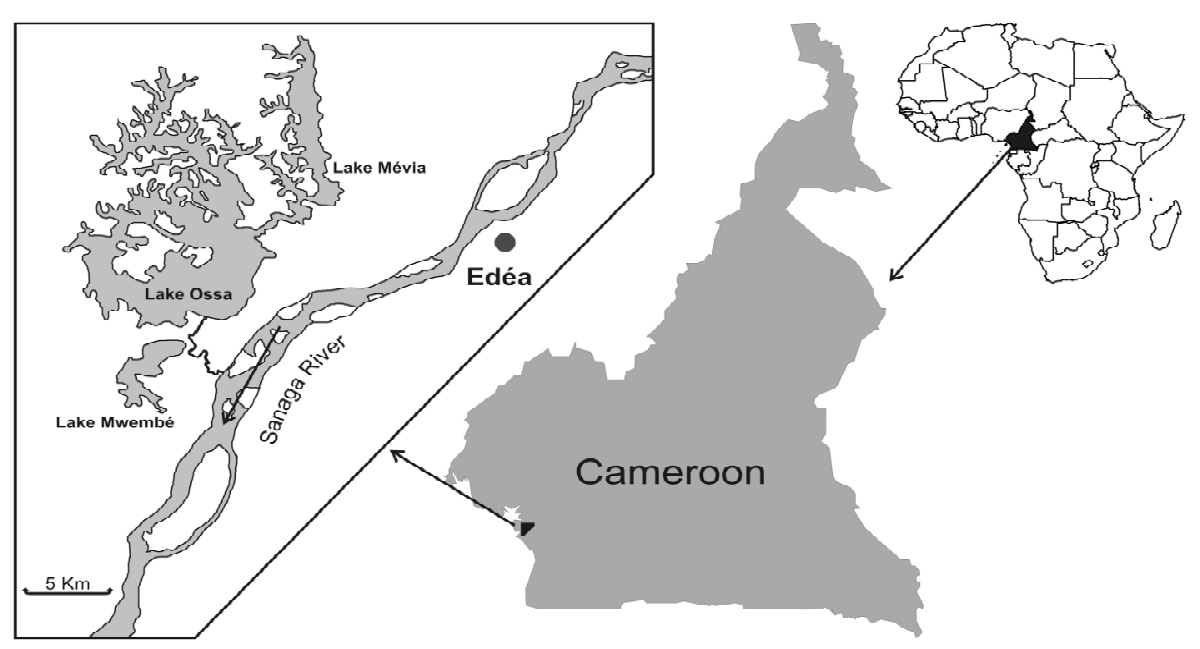

Figure 1 : Location géographique et hydrographie du Lac Ossa (d'après Wirmann et al., 2001, modifié).

Pêche, conditionnement et transport des poissons : Les spécimens de poissons ont été pêchés au filet maillant. Dès leur sortie de l'eau, les poissons ont été fixés par immersion dans une solution de formol à $10 \%$ contenue dans un bocal afin d'éviter de décrocher les monogènes à cause d'une sécrétion abondante postmortem du mucus (Bilong Bilong, 1995). Après la mort du poisson, une incision a été réalisée sur l'abdomen pour permettre une bonne conservation des viscères. Les poissons ainsi traités ont été transportés au laboratoire pour des analyses ultérieures.

Collecte des données : Au laboratoire, les huit (8) arcs branchiaux ont été détachés après deux sections dorsale et ventrale, puis introduits séparément dans des boîtes de Pétri contenant de l'eau de robinet et marquées chacune du numéro de l'arc (I à IV dans le sens antéropostérieur) et de la lettre $G$ ou $D$ selon qu'il s'agissait du côté gauche ou droit du poisson. Quatre niveaux de perception ont été 
retenus dans cette analyse : la taille de l'hôte (longueur standard); le côté de l'hôte; l'arc branchial ou l'holobranchie (gradient transversal de Rohde, 1977) ; le secteur (gradient longitudinal de Rohde, 1977), chaque holobranchie était divisée dorso- ventralement en 3 parties formée chacune d'un même nombre de filaments branchiaux (fig. 2). Dans cette étude, l'hémibranchie et la zone n'ont pas été considérées car chez C. auratus, les charges parasitaires (abondances et intensités moyennes étant faibles ou très faibles Bilong Bilong et Njiné, 1998).

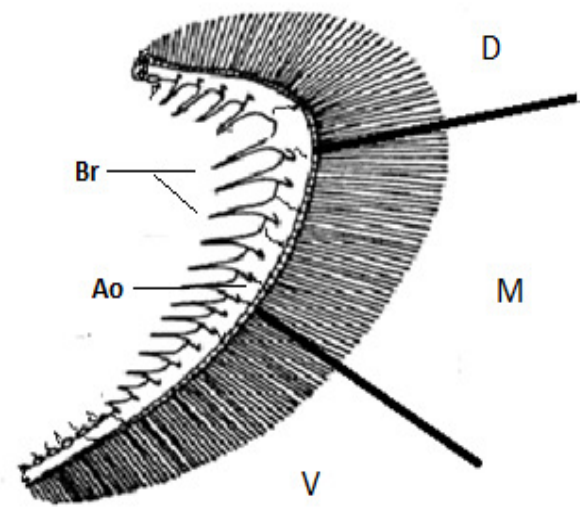

Figure 2 : délimitations de l'arc branchial de Chrysichthys auratus (Olivia et Lique ; 1998, modifié) Légende de la figure $2: A O:$ Arc osseux ; $B r$ :Branchiospines $D:$ secteur dorsal $; M:$ secteur médian $: V:$ secteur ventral

Terminologie : Dans le cadre de cette étude, les termes prévalence, abondance et intensité sont utilisés selon Margolis et al., 1982), ceux de xénopopulation et xénocommunauté conformément à Combes (1995). Le taux d'occupation désigne le pourcentage d'un côté, d'un arc ou d'un secteur, hébergeant une espèce parasite précise. La charge parasitaire moyenne correspond au nombre d'individus parasites d'une espèce donnée divisé par celui des côtés, d'arcs, des secteurs ou des zones examinés. La caractérisation des parasites en espèces

\section{RESULTATS}

Distribution de la xénocommunauté parasitaire dans la population d'hôtes : Un total de 50 poissons a été examiné. La sex-ratio était biaisée en faveur des femelles, selon un rapport de 0,85 (23 males et 27 femelles). Deux espèces de Monogènes y ont été récoltées, réparties comme suit: 1673 spécimens de Protoancylodiscoïdes combesi Bassock Bayiha, Nack et Pariselle, 2016, correspondant à une intensité moyenne de 34,14 (intensité maximale : 177), pour une prévalence de $71,4 \% ; 671$ Protoancylodiscoïdes auratum Bassock Bayiha, Nack et Pariselle, 2016, ce qui correspond à une intensité moyenne de 16,37 (intensité maximale : 55 ), pour une prévalence de $28,6 \%$. Dix neuf individus ( $38 \%$ principale, secondaire et rare (satellite) est faite selon Kooskivaara et Valtonen (1991)

Analyse statistique : Le test de $\chi^{2}$ a permis de comparer les taux d'occupation. Le test $\mathrm{K}$ de Kruskall wallis a permis de comparer les moyennes de plusieurs charges parasitaires lorsque les effectifs étaient inférieurs à 30 . Le test $\mathrm{T}$ de Student a permis de comparer les charges moyennes de deux échantillons pour des effectifs supérieurs à 30 . Les charges parasitaires moyennes ont été exprimées avec leurs erreurs standards. Pour toutes les analyses, le seuil de sécurité de $95 \%$ a été retenu.

de la xénopopulation) ont arboré 1795 monogènes (76,60\% de l'effectif total). Ces deux parasites montrent donc une distribution agrégative ( $\mathrm{S}^{2}>\mathrm{CP}, \mathrm{Tab}$. II et III). $P$. combesi et $P$. auratum sont des parasites fréquents chez leur hôte (Prévalence $>50 \%$ ). Leur intensité moyenne est faible $(10<\operatorname{Im} \leq 50)$ (Tab.1). Aucune différence significative liée au sexe de l'hôte n'a été notée pour les deux helminthes, aussi bien en ce qui concerne la prévalence $\left(P>0,05 ; T^{2}=0,007\right.$ et 0,07 respectivement pour $P$. combesi et $P$. auratum $\mathrm{ddl}=1$ ) qu'avec les abondances moyennes ( $P>0,05 ; t=0,92$ et 0,62 respectivement, $\mathrm{ddl}=48$ ). 

Paperna, parasites branchiaux de Chrysichthys auratus dans le Lac Ossa (Littoral -Cameroun)

Distribution du parasitisme en fonction de la longueur standard de l'hôte : Pour les deux espèces de Monogènes, aucune différence significative liée la taille n'a été notée, notamment lorsqu'on considère la prévalence $\left(P>0,05 ; T^{2}=1,26\right.$ et 0,19 respectivement $\mathrm{ddl}=3$ ) ou les abondances $(P>0,05 ; K=5,203$ et 0,83 respectivement ddl=3) chez l'hôte (Tab. 2).

Tableau 1 : Distribution des hôtes et biomasse de $P$. combesi et $P$. auratum en fonction du niveau d'infestation

\begin{tabular}{|c|c|c|c|c|c|c|}
\hline \multicolumn{2}{|l|}{ Niveau d'infestation } & $\operatorname{Nul}(0)$ & $\begin{array}{l}\text { Très faible } \\
1<C P \leq 10\end{array}$ & $\begin{array}{l}\text { Faible } \\
10<C P \leq 50\end{array}$ & $\begin{array}{l}\text { Moyen } \\
50<\mathrm{CP} \leq 100\end{array}$ & $\begin{array}{l}\text { Elevé } \\
C P>100\end{array}$ \\
\hline \multirow[t]{2}{*}{ Population d'hôtes } & Effectif & 1 & 11 & 19 & 12 & 7 \\
\hline & \%tage & 2 & 22 & 38 & 24 & 14 \\
\hline \multirow{2}{*}{$\begin{array}{l}\text { Xénocommunauté } \\
\text { parasitaire }\end{array}$} & Effectif & 0 & 61 & 488 & 820 & 975 \\
\hline & \%tage & 0 & 2,6 & 20,8 & 35 & 41,6 \\
\hline
\end{tabular}

$\%$ tage $=$ Pourcentage $; c c p=$ charge parasitaire

Tableau 2 : Charges parasitaires et taux d'occupation (\%) de $P$. combesi et $P$. auratum en fonction des classes de tailles de C. auratus

\begin{tabular}{|l|c|c|c|c|}
\hline \multirow{2}{*}{ Classe de taille } & \multicolumn{3}{|c|}{ Espèce parasite } \\
\cline { 2 - 5 } & \multicolumn{2}{|c|}{$\boldsymbol{P . c o m b e s i}$} & \multicolumn{2}{c|}{$\boldsymbol{P . ~ a u r a t u m}$} \\
\cline { 2 - 5 } & Taux d'occupation & Charge moyenne & $\begin{array}{c}\text { Taux } \\
\text { d'occupation }\end{array}$ & $\begin{array}{c}\text { Charge } \\
\text { moyenne }\end{array}$ \\
\hline$<120$ & 100 & 5 & 100 & 20 \\
\hline$[120-140[$ & 97 & $26,29 \pm 27,79$ & 86 & $12,57 \pm 15,07$ \\
\hline$[140-160[$ & 100 & $36,42 \pm 36,93$ & 80 & $14,11 \pm 16,05$ \\
\hline$[160-180[$ & 100 & $120 \pm 57$ & 100 & $15,5 \pm 10,50$ \\
\hline
\end{tabular}

Occupation du côté de l'hôte: Les résultats des analyses statistiques sur la distribution des taux d'occupation et la répartition des charges parasitaires suggèrent que $P$. combesi et $P$. auratum exploitent les deux côtés de l'hôte de la même manière. $\left(P>0,05, X^{2}\right.$ vaut 0,11 et 0,48 pour les taux d'occupation, et tégale 1,05 et 0,13 pour les charges parasitaires moyennes respectivement (Tab. 3).

Tableau 3: Charges parasitaires moyennes et taux d'occupation (\%) de $P$. combesiet $P$. auratum en fonction du côté de C. auratus.

\begin{tabular}{|l|c|c|c|c|}
\hline \multirow{3}{*}{ Indices parasitaires } & \multicolumn{4}{|c|}{ Espèces parasites } \\
\cline { 2 - 5 } & \multicolumn{2}{|c|}{$\boldsymbol{P}$. combesi } & \multicolumn{2}{c|}{$\boldsymbol{P}$. auratum } \\
\cline { 2 - 5 } & Côté gauche & Côté droit & Côté gauche & Côté droit \\
\hline Charges parasitaires & $15,34 \pm 16,63$ & $18,12 \pm 24,91$ & $6,76 \pm 8,32$ & $6,66 \pm 7,82$ \\
\hline Taux d'occupation & 96 & 98 & 50 & 46 \\
\hline
\end{tabular}

Occupation des arcs branchiaux: Les données obtenues à l'issue des analyses statistiques montrent que chez l'hôte étudiée, $P$. combesi et $P$. auratum préfèrent les arcs branchiaux I, II et III par rapport à l'arc IV $(P<0,0$ $5 ; X^{2}=1,36$ et 1,08 respectivement ddl=3). L'analyse des charges parasitaires montrent pour les abondances moyennes (Tableau 4) que $P$. combesi est plus présent sur les arcs branchiaux I, II, et III par rapport à l'arc IV
( $P<0,05 \mathrm{~K}=11,83 \mathrm{ddl}=3$ ) alors que $P$. combesi exploite les quatre arcs de manière similaire $(P>0,05 ; K=5,193$; $\mathrm{ddl}=3$ ). Les profils de cette occupation sont $: \mathrm{I}=\| \mathrm{I}=\mathrm{III}<$ IV pour la prévalence des deux parasites puis $\mathbf{I}=\| \mathbf{I}=\mathbf{I I}<$ IV et I = II = III = IV respectivement pour $P$. combesi et $p$. auratum.

Tableau 4 : Charges parasitaires et taux d'occupation (\%) de $P$. combesi et $P$. auratum en fonction de l'arc branchial de C. auratus 
Nack et al., J. Appl. Biosci. 2018 Distribution spatiale de deux monogènes du genre Protoancylodiscoides Paperna, parasites branchiaux de Chrysichthys auratus dans le Lac Ossa (Littoral -Cameroun)

\begin{tabular}{|l|c|c|c|c|}
\hline \multirow{2}{*}{ Arc branchial } & \multicolumn{4}{|c|}{ Espèce parasite } \\
\cline { 2 - 5 } & \multicolumn{2}{|c|}{$\boldsymbol{P .}$ auratum } & \multicolumn{2}{c|}{ P. combesi } \\
\cline { 2 - 5 } & Charge moyenne & Taux d'occupation & Charge moyenne & Taux d'occupation \\
\hline I & $13,20 \pm 15,66$ & 94 & $4,86 \pm 6,11$ & 76 \\
\hline II & $9,62 \pm 12,36$ & 82 & $4,36 \pm 35,83$ & 65 \\
\hline III & $5,96 \pm 6,93$ & 98 & $2,34 \pm 3,03$ & 62 \\
\hline IV & $4,68 \pm 5,72$ & 74 & $1,66 \pm 2,63$ & 58 \\
\hline
\end{tabular}

Occupation des secteurs: Les charges parasitaires moyennes (CM) des deux monogènes étant faibles (Tab. 2), l'analyse du parasitisme par secteur a été faite en considérant simultanément les quatre arcs branchiaux. Les résultats obtenus révèlent que pour $P$. auratum et $P$. combesi, le secteur médian est préféré par rapport aux secteurs dorsal et ventral $\left(P<0,05 ; T^{2}=0,11\right.$ et 2,93 respectivement et $d d l=2$, Tab. 5). La comparaison des charges parasitaires intersectorielles montre que les trois secteurs sont sollicités de manière équivalente par les deux monogènes ( $P>0,05 ; \quad K=1,351$ et 1,363 respectivement; $\mathrm{ddl}=2$ )

Tableau 5 : Charges parasitaires moyennes et taux d'occupation (\%) sectorielle de $P$. auratum et $P$. combesi sur les arcs branchiaux de C. nigrodigitatus

\begin{tabular}{|l|c|c|c|c|}
\hline \multirow{3}{*}{ Secteur branchial } & \multicolumn{4}{|c|}{ Espèce parasite } \\
\cline { 2 - 5 } & \multicolumn{2}{|c|}{$\boldsymbol{P .}$ auratum } & \multicolumn{2}{c|}{ P. combesi } \\
\cline { 2 - 5 } & Charge moyenne & Taux d'occupation & Charge moyenne & Taux d'occupation \\
\hline Dorsal & $7,38 \pm 5,31$ & 91,50 & $3,75 \pm 5,28$ & 60,00 \\
\hline Médian & $11,26 \pm 13$ & 98,30 & $4,40 \pm 5,55$ & 79,00 \\
\hline Ventral & $10,42 \pm 9,46$ & 90,00 & $4,42 \pm 5,33$ & 70,00 \\
\hline
\end{tabular}

\section{DISCUSSION ET CONCLUSION}

Dans le Lac Ossa, $P$. combesi est une espèce fréquentes alors que $P$. auratum est une espèce moins fréquente puisse que leur prévalence sont respectivement $71,4 \%$ et 28,6\%ils sont donc considérées comme des espèces fréquentes (Kooskivaara et Valtonen, 1992). Par contre, leurs abondances respectives situées entre 10 et 50 sont faibles (Bilong Bilong et Njiné, 1998). Une distribution agrégative des deux monogènes a été notée au cours de cette étude. Amed et al., 2003 ont aussi noté une distribution agrégative de Protoancylodiscoides mansourensis chez le même hôte en Egypte. De même, Tombi et al. (2014), Tombi et Bilong Bilong (2004) ont également constaté une distribution agrégative de Cichlidogyrus thurstonae, C. halli, C. tilapiae, et Scutogyrus longicornis parasites branchiaux de 0 . niloticus d'une part et Dactylogyrus bopeleti, $D$. insolitus, D. maillardi, D. simplex parasites branchiaux de Barbus martorelli d'autre part. Des observations similaires ont été faites par d'autres auteurs tels que Lo et Morand (2000), Öztürk et altunel (2006). Plusieurs hypothèses expliquent la distribution agrégative des monogènes parasites branchiaux. Par exemple, Ahmed et al., 2003 C. auratus vit en bandes dans des habitats spécifiques en profondeur proche de la boue proche des œuf et des oncomiracidia, par conséquent certains individus sont plus exposés que d'autres. Combes (1995) pense qu'une distribution agrégative des parasites illustrent l'hétérogénéité dans le système hôte/population de parasites. Pour Kennedy (1977) la distribution agrégative accroit l'opportunité pour un parasite de rencontrer un partenaire en vue de la reproduction. Ces trois hypothèses peuvent expliquer la distribution agrégative des deux monogènes étudiés dans ce travail. Les charges parasitaires de $P$. combesi et $P$. auratum sont faibles. Plusieurs auteurs ont déjà obtenus des charges parasitaires faibles chez différentes espèces hôtes. C'est par exemple le cas de Tombi et al. (2014) pour Cichlidogyrus thurstonae, C. halli, C. tilapiae et Scutogyrus longicornis, parasites de Oreochromis niloticus, Tombi et Bilong Bilong (2004) pour les espèces de Dactylogyrus, parasites de Barbus martorelli et de Koyun (2011 pour Dactylogyrus minutus et $D$. onchoratus sur les branchies de Carassius carassius. Ce modèle de distribution semble différent dans les systèmes d'élévage à cause du confinement des poissons (Ergens, 1983, Obiekezié D'après Combes (1995), les faibles ou très faibles charges parasitaires sont dues au faible recrutement des oncomiracidia associé à la mortalité 


\section{Nack et al., J. Appl. Biosci. 2018 Distribution spatiale de deux monogènes du genre Protoancylodiscoides}

Paperna, parasites branchiaux de Chrysichthys auratus dans le Lac Ossa (Littoral -Cameroun)

naturelle qui empêche que les populations de monogènes atteignent jamais un seuil dangereux. Au cours de cette étude, aucune préférence des deux parasites en fonction du sexe de l'hôte n'a été observée. Cette observation concorde celle de Tombi et Bilong Bilong (2004) avec Barbus martorelli. En Afrique du Sud, Le Roux et al. (2011) ont fait le même constat pour Cichlidogyrus philander parasite branchial de Pseudocrenilabrus philander. Cette observation renforce l'idée que très peu de parasites ont une préférence vis-à-vis du sexe de leur hôte (Rohde, 1993). Mais au Cameroun Tombi et al. (2014) ont trouvé que Scutogyrus longicornis se concentre plus chez les mâles par rapport aux femelles de 0 . niloticus. D'autres études ont également montré cette tendance des monogènes à avoir une préférence pour les individus d'un sexe donné (Blahoua et al., 2009 ; Ibrahim, 2012). Dans le present travail, la distribution de $P$. combes et $P$. auratum est identique sur les arcs branchiaux des côtés droit et gauche de $C$. auratus. Ahmed et al., 2003, chez le même hôte, Tombi et al. (2014), ainsi que Nack et al. (2010) ne notent aucune préférence respectivement chez les 0 . niloticus et Clarias camerunensis au Cameroun. De même, Soyul et al. (2013) ne signalent aucune différence dans la distribution de Pseudodactilogyrus anguillae entre les cotés droit et gauche de Anguilla anguilla. Des observations similaires sont rapportées par Gutiérrez \& Martorelli (1994) ; Dzika (1999); Turgut et al., (2006); Tombi et al., (2010). Hendrix (1990), au contraire, observe une distribution asymétrique du monogène Bothithrema bothi sur les branchies de Scophtalmus aquosus. Rohde (1993) explique la préférence d'un côté donné de l'hôte par l'anatomie asymétrique des parasites et de leur hôte. La distribution symétrique des individus de $P$. combesi et $P$. auratum est donc liée à la symétrie bilatérale des poissons du genre Chrysichthys et à celle des monogènes du genre Protoancylodiscoïdes. Les résultats de cette étude indiquent par ailleurs que chez $C$. auratus, l'exploitation des quatre arcs branchiaux par $P$. combesi et $P$. auratum diminue dans le sens antéro-paostérieur (de l'arc I à l'arc IV), avec une tendance à l'homogénéité dans la répartition de la charge parasitaire pour $P$. auratum. Ce résultat va dans le même sens que celui de Ahmed et al., 2003 chez le même hôte et de Tombi et al. (2014) qui chez $O$. niloticus, ont montré pour $P$. mansourensis, Cichlidogyrus thurstonae et $C$. tilapiae que la prévalence et l'intensité moyenne décroissent de l'arc I à l'arc IV et, par ailleurs, que Scutogyrus longicornis était plus fréquent sur l'arc I et plus rare sur l'arc IV. De même, Rubio-Godoy (2008) a signalé que la prévalence de Discocotyle sagittata sur les quatre arcs branchiaux de
Oncorhynchus mykiss diminue de l'arc I à l'arc IV. Beaucoup de cas de préférence des arcs branchiaux par les monogènes sont connus. Dzika (1999) a signalé que Pseudodactylogyrus anguillae se fixe plus sur les arcs baranchiaux médians (II et III) de Anguilla anguilla. C'est aussi le cas de Dactylogyrus nanus, $D$. micracanthus, $D$. suecicus et $D$. similis chez le Cyprinidae Rutilus rutilus (Bagge et Valtonen, 1999). Stavrescu-Bedivan et al. (2008) ont quant à eux observé que l'arc I de Barbus meridionalis était plus infesté par le monogène Paradiplozoon tissae.. A l' opposé Nack et al. (2010) ont constaté que Quadriacanthus sp. et Birgiellus kellensis avaient des taux d'occupation et des charges parasitaires moyens élevées sur l'arc branchial IV par rapport à l'arc l. Les travaux de Koskivaara et al. (1991) indiquent en outre que Dactylogyrus fallax colonise les deux premiers arcs antérieurs de Rutilus rutilus (. De nombreuses hypothèses ont été formulées pour expliquer le choix de certains arcs branchiaux par les monogènes. Certains auteurs pensent que les arcs médians sont souvent plus sollicités pour deux principales raisons : ils sont traversés par un grand volume d'eau et le courant respiratoire d'eau y est important ((Gutiérrez et martorelli, 1994 ; Lo et Morand, 2001). Pour Buchmann (1989), Koskivaara et Valtonen (1991), ces arcs médians sont plus infestés parce qu'ils offrent une grande surface d'attache aux parasites. Au Cameroun, Bilong Bilong (1995); Tombi et Bilong Bilong (2004) considèrent la synergie de deux facteurs, hétérogénéité du système branchial et modèle d'écoulement du courant d'eau respiratoire, pour expliquer les abondances parasitaires sur les arcs branchiaux. D'autres auteurs ont mentionné les facteurs comme la charge parasitaire pour expliquer le choix des arcs branchiaux (Lo et Morand, 2000). Pour Koskivaara et Valtonen (1992), ainsi que pour Gutiérrez et Martorelli (1994), l'immunité de l'hôte pourrait aussi influencer la sélection des microhabitats. Dans le present travail, la répartition de $P$. auratum et $P$. combesi pourrait s'expliquer par le mode d'infestation par voie buccale des oncomiracidia des monopisthocotylea, qui atteignent d'abord l'arc I avant de progresser vers les arcs.postérieurs II, III et IV. Dans cette étude, l'analyse longitudinale (ou sectorielle) a montré, sur la base de la répartition des taux d'occupation, que $P$. auratum et $P$. combesi préfèrent le secteur médian par rapport aux deux autres secteurs. Par contre, aucune différence significative n'a été notée quant aux charges parasitaires intersectorielles. Nack et al. (2010) ont constaté que Quadriacanthus sp. et Birgiellus kellensis se fixaient préférentiellement sur le secteur médian. Des observations similaires ont été faites par Hendrix (1990), 
par Bilong-Bilong (1995), Gerasev et Staravoitov (1988), et par Yang et al. (2006). Pour tous ces auteurs, les secteurs médiodorsal, médian et médioventral $\left(\mathrm{S}_{2}, \mathrm{~S}_{3}, \mathrm{~S}_{4}\right)$ sont plus sollicités par les parasites. D'autres études ont cependant suggéré que certains monogènes aiment les secteurs dorsal S1 ou ventral S5 (Buchmann, 1993; Yang et al., 2006; Madhi et Belghyti, 2006). Lo et Morand (2001) et Tombi et al. (2014) n'ont par contre établi aucune préférence sectorielle quant à la fixation des monogènes. Le choix des secteurs branchiaux par les monogènes parasites a plusieurs interprétations. Bilong Bilong (1995) pense que certaines espèces s'attachent essentiellement sur les secteurs (médians par exemple) qui sont plus exposés au courant ventilatoire. Pour Bilong Bilong (1995) et Tombi et al. (2010), les facteurs biotiques comme la forme et la taille des pièces sclérifiées du hapteur joueraient un rôle important dans le choix des sites de fixation. Les monogènes armés de petits crochets se fixeraient préférentiellement sur la region basale des filaments branchiaux pour s'abriter $\mathrm{du}$ courant fort (Bilong Bilong, 1995 ; Nack et al. 2010). Par contre Birgiellus kellensis, aux crochets relativement grands et au corps trapu, se fixerait prioritairement sur la zone basale afin d'éviter d'être emporté par le courant ventilatoire. D'autres auteurs pensent que la préférence de certains sites par les monogènes serait liée à la compétition intra et interspécifique. Les travaux de Ramassamy et al. (1985) sur les poissons Téléostéens du genre Scomberoides dans la côte sud de l'Inde, ont montré que les monogènes Vallisa indica, Allodiscocotyla chorinema et Heterata chorineme (Monopisthocotylea) montrent une préférence de site et que cette préférence est densité parasitaire dépendante. Ils retiennent donc que la compétition intra et interspécifique joue un rôle important dans l'occupation de l'espace branchial et rapportent que des facteurs intrinsèques comme la reproduction restreignent les monogènes à certains sites.

\section{REMERCIEMENTS}

Les auteurs remercient Dr BITJA NYOM Arnold Roger de l'Institut des Sciences Halieutiques de l'Université de

\section{REFERENCES BIBLIOGRAPHIQUES}

Ahmed M, El-Naggar, Enayat S, Reda. 2003. Distribution of Protoancylodiscoides mansourensis ElNaggar, 1987, a monogenean gill parasite from the long catfish Chrysichthys auratus Geoffroy, 1809. Egypt. Journal of Aquatic Biotechnology and Fisheries, 7(4): 331 - 357.
Par contre, d'après Buchmann (1989), Koskivaara et Valtonen (1991) et Price (1980), tant qu'il y a assez d'espace et de ressources, les interactions interspécifiques sont rares. Allant dans le même sens, Rohde (1979) rapporte par ailleurs que les biotopes occupés par les parasites n'atteignent pas souvent leur capacité maximale de remplissage, de ce fait l'exclusion compétitive ne peut avoir lieu. L'intervention d'un facteur abiotique (le courant ventilatoire de l'eau à travers les branchies) jouerait donc aussi un rôle important dans le choix du site de fixation par les monogènes. Par exemple, Les monogènes munis de petits crochets s'abritent des courants forts en se fixant sur la zone basale filamenteuse, induisant une réaction tissulaire renforçant leur adhésion à l'hôte; c'est le cas de Pseudodactylogyrus bini chez Anguilla anguilla (Dzika, 1999), de Onchobdella aframae chez Hemichromis fasciatus, alors que les Cichlidogyrus plus robustes occupent les zones distales (Bilong Bilong, 1995). Dans le présent travail, la fixation de $P$. combesi et de $P$. auratum sur les différents secteurs serait le fait du hasard, à cause leur faible charges et du fait que les crochets des deux espèces ont des formes et les tailles similaires (Bassock et al., 2016). Les informations fournies par cette étude ont deux applications directes : pour les pisciculteurs, elles permettent de savoir que dans le Lac Ossa et le bassin de la Sanaga, $C$. auratus est parasité par deux espèces de monogènes avec des charges parasitaires faibles et très faibles, donc tout essaie de domestication devrait être fait dans un environnement peu modifié et précédée d'un déparasitage des poissons. Pour les scientifiques fondamentalistes, l'étude permet de connaitre le modèle de fixation des espèces du genre Protoancylodiscoïdes qui est antéro-postérieur et que dans cette situation de faible intensité parasitaire la compétition n'intervient pas dans le choix de site de fixation des monogènes.

Douala (Cameroun) qui a identifié les poissons hotes examinés au cours de cette étude.

Bagge AM and Valtonen ET. 1999. Development of monogenean communities on the gill of roach fry (Rutilus rutilus). Parasitology. 118: 479- 487.

Bassock Bayiha ED, Nack J, Pariselle A, Bilong Bilong CF. 2016. Two new species of gill parasites assigned to Protoancylodiscoides (Monogenea, Ancyrocephalidae) from Chrysichthys spp. 
(Siluriformes, Claroteidae) in River Sanaga (Cameroon). Zootaxa 4170(1): 178-186.

Bilong Bilong CF. 1995. Les monogènes parasites d'eau douce du Cameroun : biodiversité et spécificité ; biologie des populations inféodées à Hemichromis fasciatus. Thèse de Doctorat d'État, Université de Yaoundé I. 341p.

Bilong Bilong CFet Njiné T. 1998. Dynamique de population de trois Monogènes parasites d'Hemichromis fasciatus Peters, 1858 dans le Lac Municipal de Yaoundé et Intérêt possible en pisciculture intense. Ann. Fac Sci; Univ. Ydé I, Série Sciences Naturelles et Vie 34(2) : 295 303.

Bilong Bilong CF et Tombi J. 2004. Hétérogénéité du système branchial de Barbus martorelli Roman, 1971 (Poisson : Cyprynidae) et modèle de croissance. Journal of Cameroon \&aAcademy of Sciences. Vol. $4 \mathrm{n}^{\circ} 3$.

Blahoua KG., N'Douba V., Tidiani K., N'Guessan K J. 2009. Variations saisonnières des indices épidémiologiques de trois monogènes parasites de Sarotherodon melanotheron (Pisces: Cichlidae) dans le lac d'Ayamé (Côte d'lvoire). Sciences \& Nature. 6(1): 39-47.

Buchmann K. 1989. Microhabitat of monogenean gill parasites on European eel (Anguilla anguilla). Folia Parasitologica. 36:321-329.

Buchmann K. 1993. Epidémiologie et contrôle des infections à Pseudodactylogyrus dans les élevages intensifs d'anguilles : données actuelles. Bulletin Français de pêche et de Pisciculture. 328 : $66-73$.

Combes C. 1995. Rencontre, identification, installation dans le cycle des métazoaires parasites. Bulletin de la Société de Zoologie Française 115 (1) : $99-105$

Combes $\mathrm{C}$ and Jourdanes J. 2003. Taxonomie écologie et évolution des métazoaires parasites taxonomy ecology and evolution of metazoan parasites. (Livre hommage à Louis Euzet) Tome I. PUP, Perpignan France. p : 161 - 201.

Dossou CT. 1985. Monogènes parasites de poissons d'eau douce au Bénin (Ouest-africain).Doctoral thesis, Université des Sciences et Techniques du Languedoc, Montpellier, France, $133 \mathrm{p}$.

Dzika E. 1999. Microhabitats of Pseudodactylogyrus anguillae and P. bini (Monogenea :

Dactylogyridae) on the gill of the large size european eel Anguilla anguilla from Lake gaj, Poland. Folia parasitologica. 46 : $33-36$.
Euzet $L$ and Combes C. 1998. The selection of habitat among the monogenea. International Journal of Parasitology. 28: $1654-1662$.

FAO. 2014. La situation mondiale des pêches et de l'aquaculture, possibilités et défis. Rome, 255p.

Gerasev PI and Staravoitov VK. 1988. Distribution of Ancyrocephalus paradoxus (Monogenea) on gills of adult pike perch Stizostedion lucioperca in the Courish Bay. Proceeding of The Zoological Institut. Leningrad. 177: 89-98.

Gutiérrez PA and Martorelli SR, 1994. Seasonality, distribution and preference sites of

Demisdospermus valenciennesi Gutirrez \& Suriano, 1992 (Monogenea: Ancyrocephalidae) in catfish. Research review in Parasitology. 54(4): 259 261.

Hendrix SS. 1990. Attachment and microhabitat of Bothritrema bothi(Monogenea). ICOPA VII, Paris, S6 A. Dynamique des populations parasites. Résumé de la communication. $1 p$.

Ibrahim MM. 2012. Variation in parasite infracommunies of Tilapia zillii in relation to some biotic and abiotic factors. International Journal of Zoological Research. 8(2): 59-70.

INS. 2013. Elevage et pêche in : Annuaire statistique du Cameroun. p: 265 - 267.

Kennedy CR. 1977. The regulation of fish parasite populations. In: G.W. Esh (Ed). Regulation of parasite populations. New York: Academic Press. 253p.

Kooskivaara M and Valtonen T. 1991. Paradiplozoon homoion (monogenea) and some others gill parasites on Roach Rutilus rutilus in Finland. Aqua fennica 21( 2): 137 - 146.

Koskivaara M and Valtonen ET. 1992. Dactylogyrus (Monogenea) communities on the gill of roach in three lakes in Central Finland. Parasitology. 104: 263-272.

Kookivaara M, Valtonen T, Vuori KM, 1991. Microhabitat distribution and coexistence of Dactylogyrus species (Monogenea) on the gill of Roach. Parasitology. 104: 273 - 281.

Koyun M. 2011. Seasonal distribution and ecology of some Dactylogyrus species infecting Alburnus alburnus and Carassius carassius (Osteichthyes: Cyprinidae) from Porsuk river, Turkey. African Journal of Biotechnology. 10(7): 1154-1159.

Le Roux LE., Avenant-Oldewage A and Walt van der FC. 2011. Aspects of the ecology of Cichlidogyrus philander collected from Pseudocrenilabrus 
Philander philander from the Padda Dam, Gauteng, South Africa. African Zoology. 46: 103116.

Lo CM, and Morand S. 2000. Spatial distribution and coexistence of monogenean gill parasites inhabiting two damselfishes from Moorea Island in French Polynesia. Journal of Helminthology. 74(4): 329-36.

Lo CM and Morand S. 2001. Gill parasites of Cephalopholis argus (Teleostei: Serranidae) from Moorea (french polynesia): site selection and coexistence. Folia Parasitologica. 48: 30-36.

Madhi YE et Belghiti D. 2006. Répartition branchiale des monogènes Gotocotylea acanthura et Pyragraphorus hollisae parasites du Pompano, Trachinotus ovatus (Pisces, Carangidae) de la côte Medhia (Marocc). Agronomie Africaine. 18(2): $117-124$.

Margolis L, Esh GW, Holmes JC, Kuris AM, Schad GA. 1982. The use of ecological terms in parasitology (report of ad hoc committee of American Society of parasitologists). Jounal of Parasitology. 68(1): 131 - 133.

Nack J., Tombi J., Bitya Nyom A, Bilong Bilong CF. 2010. Sites de fixation de deux monogènes Dactylogyridae parasites branchiaux de Clarias camerunensis : évidence sur le mode d'infestation par les Monopisthocotylea. Journal of Applied Biosciences. 33: 2076-2083.

Obiekezie A I. 1991. The principal pathogens and diseases of cultured fishes in Nigeria. In : IFS (Eds). Aquaculture in Africa. p: 197-207.

Obiekezie A.I., Muller H, Anders K. 1988. Diseases of the african estuarine catfish Chrysichthys nigrodigitatus (Lacépède) from the Cross River estuary, Nigeria. Journal of fish Biology 32: 207221.

Okaeme A.N., Obiekezie, A.I., Lehman, J., Antai, E.E, Madu, CT. 1988. Parasites and diseases of cultured fish of Lake Kainji area Nigeria. Journal of Fish Biology. 32: 479-481.

Olivia ME and Luque JL. 1998. Distribution pattern of Microcotyle nemadactylus (Monogenea) on gill filaments of Cheiloductylus variegates (Teleostei). Memory of The Institute of Oswaldo Cruz, Rio de Janeiro, 93(4): 477 - 478.

Öztürk MO and Altunel FN. 2006. Occurrence of Dactylogyrus infection linked to seasonal changes and host fish size on four cyprinid fishes in lake Manyas, Turkey. Acta zoologica Hungarica. 52(4): 407-415.
Paperna I. 1969. Monogenetic trematodes of the fish of the Volta Bassin and south Ghana. Bulletin de I'Institut française d'Afrique Noire, Série A,XXXI, 840-880.

Price PW. 1980. Evolutionary Biology of parasites, Princeton University press, Princeton.

Ramasamy P, Ramalingam K, Hanna REB, Halton DW (1985). Microhabitats of gill arasites (Monogenea and Copepoda) of teleosts (Scomberoides spp.). International Journal of Parasitology. 15(4): 385-397.

Rohde K. 1977. A non competitive mechanism responsible for restricting niches. Zoologischer Enzeiger. 199 (3/4): 164-172.

Rohde K. 1979. A critical evaluation of intrinsic factors responsible for niche restriction in parasites. American Naturalist. 114: 648 - 671.

Rohde K. 1993. Ecology of marine parasites. An introduction to marine parasitology. $2^{\mathrm{e}}$

Ed. CAB INTERNATIONAL, Wallinford. Oxon : 298p.

Rubio-Godoy M. 2008. Microhabitat selection of Discocotyle sagittata (Monogenea : Polyopisthocotylea) in farmed rainbow trout. Folia Parasitologica. 55: 270-276.

Stavrescu-Bedivan M M and Aioanei FT., 2008. Aspects of branchial parasitism in Barbus meridionalis Petenyi Heckel, 1847(Teleostei : Cyprinidae). Bulletin UASVM, Veterinary Medecine. 65(2): 57-90.

Stiassny MLJ, Teugels GG, Hopkins CD. 2007. Poissons d'eaux et saumâtres de basse Guinée, ouest de I'Afrique centrale. Volume I. IRD, MnHn, MRAC, Paris, $800 \mathrm{p}$.

Soylu E., Çolak SO., Erdogan F., Erdogan M, Tektas N. 2013. Microhabitat distribution of Pseudodactylogyrus anguillae (Monogenea), Ergasilus gibbus and Ergasilus lizae (Copepoda) on the gills of European Eels (Anguilla anguilla, L.) Acta zoologica Bulgarica. 65(2): 251-257.

Tombi J. and Bilong Bilong CF. 2004. Distribution of gill parasites of the freshwater fish Barbus martorelli Roman, 1971 (Teleostei : Cyprinidae) and tendency to inverse intensity evolution between Myxosporidia and Monogenea as a function of the host age. Revue d'Elevage et de Médecine Vétérinaire de Pays Tropicaux. 57(1-2): 71-76.

Tombi J., Akoumba JF, Bilong Bilong CF. 2014. The monogenean community on the gills of Oreochromis niloticus from Melen Fish station in Yaoundé, Cameroun. International Journal of Modern Biological Research 2: 16 - 23. 
Turgut E., Shinn A, Wootten R. 2006. Spatial distribution of Dactylogyrus (Monogenean) on the gills of the host fish. Turkish Journal of Fisheries and Aquatic Sciences. 6: 93-98.

Wirmann D. 1992. Le Lac Ossa: une monographie preliminaire. Revue Geographique du Cameroun. XI(1) : $27-38$.

Worldfish Center. 2005. Le poisson et la sécurité alimentaire en Afrique. WorldFish Center Penang (Malaisie). 11p.

Yang T, Liu J. Gibson DI, Dang A. 2006. Spatial distribution of two species of monogenans on the gills of Siamus fuscecens (Houhyn) and their seasonal dynamics in caged versus wild-Caugth hosts. Journal of Parasitology. 92: 933-940. 